\title{
FOREWORD
}

\section{Special Section on Foundations of Computer Science}

We are very happy to publish this special section on Foundations of Computer Science. This is the fourth section on this title. The main goal of this special section is to review and promote recent progress in the field of foundations of computer science.

The special section includes nine full papers, which cover a variety of topics in the area such as approximation algorithms, finite automata, formal language, graph algorithms, graph theory, optimizing algorithms, parallel computing and quantum computing. These nine papers were selected among eighteen submissions through careful review.

As the Guest Editor of this special section, I would like to express my sincere thanks to all authors for their contributions. I greatly appreciate the voluntary work of all reviewers and also the remarkable efforts of the members of the Editorial Committee listed below.

Editorial Committee Members

Akihiro Fujiwara (Kyushu Institute of Technology)

Chuzo Iwamoto (Hiroshima University)

Yuichi Kaji (Nara Institute of Science and Technology)

Takuya Kida (Hokkaido University)

Yosuke Kikuchi (Tsuyama National College of Technology)

Mitsuo Motoki (JAIST)

Yoshio Okamoto (Toyohashi University of Technology)

Masahiko Sakai (Nagoya University)

Shinichi Shimozono (Kyushu Institute of Technology)

Yasuhiko Takenaga (The University of Electro-Communications)

Eiji Takimoto (Tohoku University)

Keisuke Tanaka (Tokyo Institute of Technology)

Yushi Uno (Osaka Prefecture University)

Shin-ichi Nakano, Guest Editor

Shin-ichi Nakano (Member) received his B.E. and M.E. degrees from Tohoku University, Sendai, Japan, in 1985 and 1987, respectively. In 1987 he joined Seiko Epson Corp. and in 1990 he joined Tohoku University. In 1992, he received D.E. degree from Tohoku University. Since 2004 he is a Professor in the Department of Computer Science, Gunma University. His research interests are graph algorithms and graph theory. He is a member of IPSJ, JSIAM, ACM and IEEE Computer Society. 